\title{
Surprising Sinc Sums and Integrals
}

\section{Robert Baillie, David Borwein, and Jonathan M. Borwein}

1. MOTIVATION AND PRELIMINARIES. We intend to show that a variety of trigonometric sums have unexpected closed forms by relating them to cognate integrals. We hope this offers a good advertisement for the possibilities of experimental mathematics, as well as providing both some entertaining examples for the classroom and a caution against over-extrapolating from seemingly compelling initial patterns.

Recall the standard convention $\operatorname{sinc}(x):=\sin (x) / x$ when $x \neq 0$ and $\operatorname{sinc}(0):=1$. It is known (see, for example, [4] and [3]) that

$$
\int_{0}^{\infty} \operatorname{sinc}(x) d x=\int_{0}^{\infty} \operatorname{sinc}^{2}(x) d x=\frac{\pi}{2},
$$

while

$$
\sum_{n=1}^{\infty} \operatorname{sinc}(n)=\sum_{n=1}^{\infty} \operatorname{sinc}^{2}(n)=\frac{\pi}{2}-\frac{1}{2}
$$

Since sinc is an even function we can remove the mysterious $-1 / 2$ from (2) to get the equivalent statement

$$
\int_{-\infty}^{\infty} \operatorname{sinc}(x) d x=\int_{-\infty}^{\infty} \operatorname{sinc}^{2}(x) d x=\sum_{n=-\infty}^{\infty} \operatorname{sinc}(n)=\sum_{n=-\infty}^{\infty} \operatorname{sinc}^{2}(n)=\pi .
$$

In the rest of the paper we do not restate this sort of equivalence and mainly use the one-sided sums and integrals which are more familiar to most readers, rather than the two-sided versions which are more natural from a Fourier analysis perspective.

Experimentation with Mathematica suggested that for $N=1,2,3,4,5$, and 6, the sum

$$
\sum_{n=1}^{\infty} \operatorname{sinc}^{N}(n)
$$

is $-1 / 2$ plus a rational multiple of $\pi$. But for $N=7$ and $N=8$, the results are completely different: Mathematica gives polynomials in $\pi$ of degree 7 and 8 respectively. For example, for $N=7$, we get

$$
-\frac{1}{2}+\frac{129423 \pi-201684 \pi^{2}+144060 \pi^{3}-54880 \pi^{4}+11760 \pi^{5}-1344 \pi^{6}+64 \pi^{7}}{46080} .
$$

These results are surprising, and we explain them below. But there's more. Further experimentation suggested that for $N=1,2,3,4,5$, and 6 (but not 7 or 8 ), we had

$$
\sum_{n=1}^{\infty} \operatorname{sinc}^{N}(n)=-\frac{1}{2}+\int_{0}^{\infty} \operatorname{sinc}^{N}(x) d x .
$$


This too was unexpected. In the integral test for infinite series, the convergence of the integral of $f(x)$ may imply the convergence of the sum of $f(n)$, but there is usually no simple relationship between the values of the sum and the corresponding integral.

We found more examples where the sum was $1 / 2$ less than the corresponding integral. In [5] and [8], it was shown that, for $N=0,1,2,3,4,5$, and 6,

$$
\int_{0}^{\infty} \prod_{k=0}^{N} \operatorname{sinc}\left(\frac{x}{2 k+1}\right) d x=\frac{\pi}{2}
$$

but that for $N=7$, the integral is just slightly less than $\pi / 2$ :

$$
\begin{aligned}
& \int_{0}^{\infty} \operatorname{sinc}(x) \operatorname{sinc}\left(\frac{x}{3}\right) \cdots \operatorname{sinc}\left(\frac{x}{15}\right) d x \\
& \quad=\pi\left(\frac{1}{2}-\frac{6879714958723010531}{935615849440640907310521750000}\right)
\end{aligned}
$$

This surprising sequence is explained by Corollary 1 of Theorem 2 in [5], which we incorporate into Theorem 2 below.

More experiments suggested that, for $N=0,1,2,3,4,5,6$, and 7, the sums were also $1 / 2$ less than the corresponding integrals:

$$
\sum_{n=1}^{\infty} \prod_{k=0}^{N} \operatorname{sinc}\left(\frac{n}{2 k+1}\right)=-\frac{1}{2}+\int_{0}^{\infty} \prod_{k=0}^{N} \operatorname{sinc}\left(\frac{x}{2 k+1}\right) d x
$$

In fact, we show in Example 1(b) below that (6) holds for every $N \leq 40248$ and fails for all larger integers! This certainly underscores the need for caution, mentioned above.

We now turn to showing that the theorems for integrals proven in [5] imply analogues for sums. Our results below use basic Fourier analysis, all of which can be found in [16] and [8], to explain the above sums, and others, and to allow us to express many such sums in closed form.

2. WHEN SUMS AND INTEGRALS AGREE. Our notation and original development in this section were based on Boas and Pollard [4], but the present more satisfactory treatment is largely due to the kind and insightful suggestions of Mark Pinsky. See also [13] for pertinent information about Fourier analysis.

Suppose that $G$ is Lebesgue integrable over $(-\infty, \infty)$ and define its Fourier transform $g$ by

$$
g(x):=\frac{1}{\sqrt{2 \pi}} \int_{-\infty}^{\infty} e^{-i u x} G(u) d u .
$$

At any point $u$ such that $G$ is of bounded variation on $[u-\delta, u+\delta]$ for some $\delta>0$ we have [16, Theorem 23] that

$$
\frac{1}{2}\{G(u+)+G(u-)\}=\lim _{T \rightarrow \infty} \frac{1}{\sqrt{2 \pi}} \int_{-T}^{T} e^{i u x} g(x) d x,
$$

where $G(u \pm)$ denotes $\lim _{x \rightarrow u^{ \pm}} G(x)$. 
Suppose, in addition, that $G(x)=0$ for $x \notin(-\alpha, \alpha)$ for some $\alpha>0$, and that $G$ is of bounded variation on $[-\delta, \delta]$ for some $\delta>0$. Then clearly

$$
g(x)=\frac{1}{\sqrt{2 \pi}} \int_{-\alpha}^{\alpha} e^{-i u x} G(u) d u,
$$

and hence, for $r=0,1,2, \ldots$, by summing the exponential, as in [15, Theorem 5.12],

$$
\sum_{n=-r}^{r} g(n)=\frac{1}{\sqrt{2 \pi}} \int_{-\alpha}^{\alpha} G(u) \frac{\sin ((r+1 / 2) u)}{\sin (u / 2)} d u .
$$

Suppose first that $0<\alpha<2 \pi$. Then

$$
\sum_{n=-r}^{r} g(n)=\frac{1}{\sqrt{2 \pi}} \int_{-\alpha}^{\alpha} G^{*}(u) \frac{\sin ((r+1 / 2) u)}{u} d u
$$

where

$$
G^{*}(u):=G(u) \frac{u}{\sin (u / 2)}
$$

Since $G^{*}$ is of bounded variation on $[-\delta, \delta]$ and Lebesgue integrable over $(-\alpha, \alpha)$, and $G^{*}(0+)=2 G(0+)$ and $G^{*}(0-)=2 G(0-)$, it follows, by a standard Jordan-type result (see the proof of Theorem 3 in [16]), that

$$
\begin{aligned}
\lim _{r \rightarrow \infty} \int_{-\alpha}^{\alpha} G^{*}(u) \frac{\sin ((r+1 / 2) u)}{u} d u & =\frac{\pi}{2}\left\{G^{*}(0+)+G^{*}(0-)\right\} \\
& =\pi\{G(0+)+G(0-)\} .
\end{aligned}
$$

The following proposition, which enables us to explain most of the above experimental identities, now follows from (7) with $u=0,(10),(11)$, and (12).

Proposition 1. If $G$ is of bounded variation on $[-\delta, \delta]$, vanishes outside $(-\alpha, \alpha)$, and is Lebesgue integrable over $(-\alpha, \alpha)$ with $0<\alpha<2 \pi$, then

$$
\lim _{r \rightarrow \infty} \sum_{n=-r}^{r} g(n)=\lim _{T \rightarrow \infty} \int_{-T}^{T} g(x) d x=\sqrt{\frac{\pi}{2}}\{G(0+)+G(0-)\} .
$$

As a simple illustration, consider the function $G$ that equals 1 in the interval $(-1,1)$ and 0 outside. The corresponding $g$ is given by $g(x)=\sqrt{2 / \pi} \operatorname{sinc}(x)$. Then (13) shows, $\operatorname{since} \operatorname{sinc}(x)$ is an even function, that

$$
1+2 \sum_{n=1}^{\infty} \operatorname{sinc}(n)=2 \int_{0}^{\infty} \operatorname{sinc}(x) d x=\pi,
$$

where the integral is an improper Riemann integral.

The prior analysis can be taken further, assuming only that $G(x)=0$ for $x \notin$ $(-\alpha, \alpha)$ for some $\alpha>0$. Suppose first that $2 \pi \leq \alpha<4 \pi$ and that $G$ is also of bounded variation on $[-2 \pi-\delta,-2 \pi+\delta]$ and $[2 \pi-\delta, 2 \pi+\delta]$. Then, by splitting the integral in (9) into three parts and making the appropriate changes of variables, we get 


$$
\begin{aligned}
\sum_{n=-r}^{r} g(n)= & \frac{1}{\sqrt{2 \pi}} \int_{-\pi}^{\pi} G(u) \frac{\sin ((r+1 / 2) u)}{\sin (u / 2)} d u \\
& +\frac{1}{\sqrt{2 \pi}} \int_{-\pi}^{\alpha-2 \pi} G(u+2 \pi) \frac{\sin ((r+1 / 2) u)}{\sin (u / 2)} d u \\
& +\frac{1}{\sqrt{2 \pi}} \int_{2 \pi-\alpha}^{\pi} G(u-2 \pi) \frac{\sin ((r+1 / 2) u)}{\sin (u / 2)} d u .
\end{aligned}
$$

Hence, from this we get as in the previous case that when $2 \pi \leq \alpha<4 \pi$,

$$
\begin{aligned}
\lim _{r \rightarrow \infty} \sum_{n=-r}^{r} g(n)= & \lim _{T \rightarrow \infty} \int_{-T}^{T} g(x) d x \\
& +\sqrt{\frac{\pi}{2}}\{G(2 \pi-)+G(2 \pi+)+G(-2 \pi-)+G(-2 \pi+)\} .
\end{aligned}
$$

This process can evidently be continued by induction to yield that, when $2 m \pi \leq \alpha<$ $2(m+1) \pi$ with $m$ a positive integer, and $G$ is of bounded variation in intervals containing the points $\pm 2 n \pi, n=0,1, \ldots, m$,

$$
\lim _{r \rightarrow \infty} \sum_{n=-r}^{r} g(n)=\lim _{T \rightarrow \infty} \int_{-T}^{T} g(x) d x+\sqrt{\frac{\pi}{2}} R_{m},
$$

where

$$
R_{m}:=\sum_{n=1}^{m}\{G(2 n \pi-)+G(2 n \pi+)+G(-2 n \pi-)+G(-2 n \pi+)\} .
$$

3. APPLICATIONS TO SINC SUMS. For an application of the above analysis let

$$
g(x):=\prod_{k=0}^{N} \operatorname{sinc}\left(a_{k} x\right)
$$

with all $a_{k}>0$, and let

$$
A_{N}:=\sum_{k=0}^{N} a_{k}
$$

The corresponding $G$ satisfying the Fourier transform equation (8) can be taken to be the function $F_{N+1}$, with differently numbered $a_{k}$, defined in Section 5 below. As shown for example in [5] and also in [12, p. 20, Entries 5.2-5.13], $G$ is positive and continuous in the interval $I_{N}:=\left(-A_{N}, A_{N}\right)$ and 0 outside the closure of $I_{N}$, and is of bounded variation on every finite interval; indeed, $G$ is absolutely continuous on $(-\infty, \infty)$ when $N \geq 1$. It therefore follows from (13) along with (14) that

$$
1+2 \sum_{n=1}^{\infty} \prod_{k=0}^{N} \operatorname{sinc}\left(a_{k} n\right)=2 \int_{0}^{\infty} \prod_{k=0}^{N} \operatorname{sinc}\left(a_{k} x\right) d x
$$

provided

$$
A_{N} \leq 2 \pi \text { when } N \geq 1 \text {, or } A_{N}<2 \pi \text { when } N=0 \text {. }
$$


The proviso is needed since (15) tells us that the left-hand side of (16) is strictly greater than the right-hand side when (17) doesn't hold, since then either (i) $N \geq 1, A_{N}>2 \pi$, and $G( \pm 2 \pi)>0$ or (ii) $N=0, A_{N} \geq 2 \pi$, and $G(2 \pi-)+G(-2 \pi+)>0$, and in either case all other terms that comprise the remainder $R_{N}$ are nonnegative. We can go further and say that when the proviso fails the constant 1 on the left-hand side of (16) has to be replaced by a constant $C<1$ that depends only on the value of $A_{N}$; unfortunately there appears to be no neat expression for $C$. We emphasize that though the case $N=0$ follows from the above analysis, neither the series nor the integral in (16) is absolutely convergent in this case. For all other values of $N$ both are absolutely convergent.

As is made clear in [4], this "sum = integral" paradigm is very general. However, as a perusal of [12] shows, there are not too many "natural" analytic $g$ for which $G$ is as required-other than powers and other relatives of the sinc function. Some nice examples are exhibited by Boas and Pollard in [4]; often they require massaging. For example, with $G(t):=\left(1+e^{i t}\right)^{\alpha}$ for $|t| \leq \pi$ and zero otherwise, and with $\alpha>-1$, they obtain a result first found by Shisha and Pollard:

$$
\sum_{n=-\infty}^{\infty}\left(\begin{array}{l}
\alpha \\
n
\end{array}\right) e^{i n t}=\int_{-\infty}^{\infty}\left(\begin{array}{l}
\alpha \\
u
\end{array}\right) e^{i t u} d u=\left(1+e^{i t}\right)^{\alpha}
$$

for $\alpha>-1,|t|<\pi$.

Additionally, however, in the case of sinc integrals, as explained in [5, Theorem 1 and Remark 1], [8], and more explicitly in [6], the right-hand term in (16) is equal to $2^{-N} V_{N} \pi / a_{0}$, where $V_{N}$ is the-necessarily rational when the $a_{k}$ are-volume of the part of the cube $[-1,1]^{N}$ between the parallel hyperplanes

$$
a_{1} x_{1}+a_{2} x_{2}+\cdots+a_{N} x_{N}=-a_{0} \text { and } a_{1} x_{1}+a_{2} x_{2}+\cdots+a_{N} x_{N}=a_{0} .
$$

Theorem 1 (Sinc Sums). One has

$$
\frac{1}{2}+\sum_{n=1}^{\infty} \prod_{k=0}^{N} \operatorname{sinc}\left(a_{k} n\right)=\int_{0}^{\infty} \prod_{k=0}^{N} \operatorname{sinc}\left(a_{k} x\right) d x=\frac{\pi}{2 a_{0}} \frac{V_{N}}{2^{N}} \leq \frac{\pi}{2 a_{0}}
$$

where the first equality holds provided

$$
A_{N}=\sum_{k=0}^{N} a_{k} \leq 2 \pi \text { when } N \geq 1 \text {, or } A_{N}<2 \pi \text { when } N=0 \text {. }
$$

The second equality needs no such restriction. Moreover (18) holds with equality throughout provided additionally that

$$
A_{N} \leq 2 a_{0}
$$

Various extensions are possible when (19) or (20) fails. The following corollary follows immediately from (18) on making the substitution $x=\tau t$ in the integral.

Corollary 1. Let $\tau$ be any positive number such that $0<\tau A_{N} \leq 2 \pi$ when $N \geq 1$, or $0<\tau A_{N}<2 \pi$ when $N=0$. Then 


$$
\frac{\tau}{2}+\tau \sum_{n=1}^{\infty} \prod_{k=0}^{N} \operatorname{sinc}\left(\tau a_{k} n\right)=\int_{0}^{\infty} \prod_{k=0}^{N} \operatorname{sinc}\left(a_{k} x\right) d x=\frac{\pi}{2 a_{0}} \frac{V_{N}}{2^{N}} \leq \frac{\pi}{2 a_{0}}
$$

In particular, (21) is independent of $\tau$ in the given interval.

When (20) fails but $A_{N-1} \leq 2 a_{0}$, as proven in [5, Corollary 1] we may specify the volume change:

Theorem 2 (First Bite). Suppose that $2 a_{k} \geq a_{N}$ for $k=0,1, \ldots, N-1$ and that $A_{N-1} \leq 2 a_{0}<A_{N}$, and $0<\tau A_{N} \leq 2 \pi$. Then

$$
\begin{aligned}
\frac{\tau}{2}+\tau \sum_{n=1}^{\infty} \prod_{k=0}^{r} \operatorname{sinc}\left(\tau a_{k} n\right) & =\int_{0}^{\infty} \prod_{k=0}^{r} \operatorname{sinc}\left(a_{k} x\right) d x \\
& =\frac{\pi}{2 a_{0}} \quad \text { for } r=0,1, \ldots, N-1
\end{aligned}
$$

while

$$
\begin{aligned}
\frac{\tau}{2}+\tau \sum_{n=1}^{\infty} \prod_{k=0}^{N} \operatorname{sinc}\left(\tau a_{k} n\right) & =\int_{0}^{\infty} \prod_{k=0}^{N} \operatorname{sinc}\left(a_{k} x\right) d x \\
& =\frac{\pi}{2 a_{0}}\left(1-\frac{\left(A_{N}-2 a_{0}\right)^{N}}{2^{N-1} N ! \prod_{k=1}^{N} a_{k}}\right)
\end{aligned}
$$

4. EXAMPLES AND EXTENSIONS. We may now explain the original discoveries:

Example 1. (a) Let $N$ be an integer and for $k=0,1, \ldots, N$, let $a_{k}:=1 /(2 k+$ 1). If $N$ is in the range $1 \leq N \leq 6$, then

$$
A_{N}=\sum_{k=0}^{N} a_{k}<2 a_{0} \text { and } A_{N}<2 \pi
$$

Hence, for each of these $N$, conditions (19) and (20) of Theorem 1 hold and so we can apply that theorem to get

$$
\frac{1}{2}+\sum_{n=1}^{\infty} \prod_{k=0}^{N} \operatorname{sinc}\left(\frac{n}{2 k+1}\right)=\int_{0}^{\infty} \prod_{k=0}^{N} \operatorname{sinc}\left(\frac{x}{2 k+1}\right) d x=\frac{\pi}{2}
$$

Now for $N=7$, condition (20) fails because

$$
\begin{aligned}
A_{N} & =1+\frac{1}{3}+\frac{1}{5}+\frac{1}{7}+\frac{1}{9}+\frac{1}{11}+\frac{1}{13}+\frac{1}{15} \\
& >2 a_{0}=2>A_{N-1}=1+\frac{1}{3}+\frac{1}{5}+\frac{1}{7}+\frac{1}{9}+\frac{1}{11}+\frac{1}{13} .
\end{aligned}
$$

However, the conditions of Theorem 2 are met, namely

$$
A_{N-1}=\frac{88069}{45045} \leq 2 a_{0}<A_{N}=\frac{91072}{45045}<2 \pi,
$$


and for each $k=0,1, \ldots, N-1$, we have $2 a_{k}>a_{N}$. Therefore, we can take $\tau=1$ and apply equation (23) of Theorem 2 to get

$$
\begin{aligned}
\frac{1}{2}+\sum_{n=1}^{\infty} \prod_{k=0}^{7} \operatorname{sinc}\left(\frac{n}{2 k+1}\right) & =\int_{0}^{\infty} \prod_{k=0}^{7} \operatorname{sinc}\left(\frac{x}{2 k+1}\right) d x \\
& =\frac{\pi}{2}\left(1-\frac{\left(\frac{91072}{45045}-2\right)^{7}}{2^{6} 7 ! \cdot \frac{1}{3} \cdot \frac{1}{5} \cdots \frac{1}{15}}\right) \\
& =\frac{\pi}{2}\left(1-\frac{6879714958723010531}{467807924720320453655260875000}\right) .
\end{aligned}
$$

(b) Let $a_{k}$ be as in part (a). If $7 \leq N \leq 40248$, then $\sum_{k=0}^{N} a_{k}<2 \pi$, so (19) holds but (20) does not. For each of these $N$, Theorem 1 tells us that

$$
\frac{1}{2}+\sum_{n=1}^{\infty} \prod_{k=0}^{N} \operatorname{sinc}\left(\frac{n}{2 k+1}\right)=\int_{0}^{\infty} \prod_{k=0}^{N} \operatorname{sinc}\left(\frac{x}{2 k+1}\right) d x<\frac{\pi}{2}
$$

For $N>40248$, the equality in the above formula fails. Indeed, equation (15) shows that

$$
\frac{1}{2}+\sum_{n=1}^{\infty} \prod_{k=0}^{N} \operatorname{sinc}\left(\frac{n}{2 k+1}\right)>\int_{0}^{\infty} \prod_{k=0}^{N} \operatorname{sinc}\left(\frac{x}{2 k+1}\right) d x
$$

since the error term is necessarily strictly positive for the requisite $G$, which was discussed at the beginning of the previous section. In a remarkable analysis based on random walks, Crandall [10] rigorously estimates that the error for $N=40249$ is minuscule: less than $10^{-226576}$. The integral is still less than $\pi / 2$ by virtue of $[\mathbf{5}$, Theorem 1]. Moreover, all subsequent errors are provably no larger than $10^{-13679}$.

(c) Let $a_{k}:=1 /(k+1)^{2}$. Because $\sum_{k=0}^{\infty} a_{k}$ converges with sum $\pi^{2} / 6$ which is both less than $2 \pi$ and less than $2 a_{0}=2$, Theorem 1 says that, for every $N \geq 0$,

$$
\frac{1}{2}+\sum_{n=1}^{\infty} \prod_{k=0}^{N} \operatorname{sinc}\left(\frac{n}{(k+1)^{2}}\right)=\int_{0}^{\infty} \prod_{k=0}^{N} \operatorname{sinc}\left(\frac{x}{(k+1)^{2}}\right) d x=\frac{\pi}{2}
$$

Thus, no matter how many factors we include, both sum and integral are unchanged! In fact, if the $a_{k}$ are the terms of any positive infinite series that converges to a sum less than or equal to $\min \left(2 \pi, 2 a_{0}\right)$, then for every $N \geq 0$,

$$
\frac{1}{2}+\sum_{n=1}^{\infty} \prod_{k=0}^{N} \operatorname{sinc}\left(a_{k} n\right)=\int_{0}^{\infty} \prod_{k=0}^{N} \operatorname{sinc}\left(a_{k} x\right) d x=\frac{\pi}{2} .
$$

Example 2. To inject a little number theory, let $p_{0}, p_{1}, p_{2} \ldots$ be the primes starting with $p_{0}=2$. Then, by Theorem 1 ,

$$
\frac{1}{2}+\sum_{n=1}^{\infty} \prod_{k=0}^{N} \operatorname{sinc}\left(\frac{n}{p_{k}}\right)=\int_{0}^{\infty} \prod_{k=0}^{N} \operatorname{sinc}\left(\frac{x}{p_{k}}\right) d x
$$

holds until $\sum_{k=0}^{N} 1 / p_{k}$ exceeds $2 \pi$. For $N=0$ and $N=1$ the value of (24) is $\pi$, but for $N=2$, Theorem 2 tells us it is $\pi(1-1 / 240)$. 
We now estimate the $N$ for which (24) ceases to hold. The sum of the reciprocals of the primes diverges slowly. In fact, $\sum\{1 / p: p . \leq x, p$ prime $\}$ is roughly $\log (\log (x))+$ $B$, where $B \sim 0.26149 \ldots$ is the Mertens constant (see, e.g., $[11$, pp. $35,79,80]$ ). In order for this sum to exceed $2 \pi, x$ must be about $y=\exp (\exp (2 \pi-B)) \sim 10^{179}$. Thus, by the Prime Number Theorem, $N \sim y / \log (y)$, which is about $10^{176}$. Thus, anyone who merely tested examples using these reciprocal prime coefficients would almost certainly never find an integer $N$ where (24) failed. This time Crandall [10] proves - without assuming the Riemann hypothesis-that the left side minus the right side of $(24)$ is less than $10^{-\left(10^{165}\right)}$, which is much less than one part in a googolplex. What makes this even more interesting is that neither side of (24) is tiny: using Theorem 4 of [10], Crandall calculates that the right side of (24) exceeds $0.686 \pi$ for all $N$. Moreover, assuming the Riemann hypothesis the upper bound on the difference reduces to $10^{-\left(10^{178}\right)}$.

Example 3. Let $1 \leq N \leq 6$, and take $a_{0}=a_{1}=\cdots=a_{N-1}=1$. Then condition (19) with $N$ replaced by $N-1$ is satisfied, so equation (18) of Theorem 1 tells us that for each $N=1,2,3,4,5$, and 6 , we have

$$
\sum_{n=1}^{\infty} \operatorname{sinc}^{N}(n)=-\frac{1}{2}+\int_{0}^{\infty} \operatorname{sinc}^{N}(x) d x .
$$

Moreover (see [5, Remark 1] and [8]), for each $N \geq 1$ the integral is an effectively computable rational multiple of $\pi$, the numerator and denominator of which are listed by Sloane in [14, Seq. A049330] and [14, Seq. A049331]. If $N=7$, then $A_{N-1}=$ $7>2 \pi$, so (19) with $N$ replaced by $N-1$ is no longer satisfied and, in this case, as Example 4 shows, the sum and the integral do not differ by $1 / 2$. Indeed, for $N \geq 7$, the sums have an entirely different quality: they are polynomials in $\pi$ of degree $N$.

We continue this discussion in the next counterexample, for which we define, for $N=1,2, \ldots$,

$$
i_{N}:=\int_{0}^{\infty} \operatorname{sinc}^{N}(x) d x, \quad s_{N}:=\sum_{n=1}^{\infty} \operatorname{sinc}^{N}(n) .
$$

\section{Example 4.}

(a) We saw in Example 3 that for $N=1,2,3,4,5$, and 6, we have $s_{N}=i_{N}-1 / 2$. By contrast $i_{7}=5887 \pi / 23040$, but Mathematica gives

$$
\begin{aligned}
s_{7}= & -\frac{1}{2}+\frac{43141}{15360} \pi-\frac{16807}{3840} \pi^{2}+\frac{2401}{768} \pi^{3} \\
& -\frac{343}{288} \pi^{4}+\frac{49}{192} \pi^{5}-\frac{7}{240} \pi^{6}+\frac{1}{720} \pi^{7} .
\end{aligned}
$$

Similarly, $i_{8}=151 \pi / 360$, and Mathematica gives

$$
s_{8}=-\frac{1}{2}+\frac{733 \pi}{210}-\frac{256 \pi^{2}}{45}+\frac{64 \pi^{3}}{15}-\frac{16 \pi^{4}}{9}+\frac{4 \pi^{5}}{9}-\frac{\pi^{6}}{15}+\frac{\pi^{7}}{180}-\frac{\pi^{8}}{5040} .
$$

Although (19) fails, we can explain these sums, and we will show how to express $s_{N}$ in closed form. 
(b) For $N \leq 6, s_{N}$ is $1 / 2$ less than a rational multiple of $\pi$. The sudden change to a polynomial in $\pi$ of degree $N$ is explained by the use of trigonometric identities and known Bernoulli polynomial evaluations of Fourier series. In general, we have the following two identities, whose proofs we leave to the reader:

$$
\sin ^{2 N+1}(n)=\frac{1}{2^{2 N}} \sum_{k=1}^{N+1}(-1)^{k+1}\left(\begin{array}{c}
2 N+1 \\
N-k+1
\end{array}\right) \sin ((2 k-1) n)
$$

and

$$
\sin ^{2 N}(n)=\frac{1}{2^{2 N-1}}\left(\frac{1}{2}\left(\begin{array}{c}
2 N \\
N
\end{array}\right)+\sum_{k=1}^{N}(-1)^{k}\left(\begin{array}{c}
2 N \\
N-k
\end{array}\right) \cos (2 k n)\right) .
$$

In particular, to compute $s_{7}$, we start with

$$
\sin ^{7}(n)=\frac{35}{64} \sin (n)-\frac{21}{64} \sin (3 n)+\frac{7}{64} \sin (5 n)-\frac{1}{64} \sin (7 n) .
$$

Now, for $0 \leq x \leq 2 \pi$,

$$
\sum_{n=1}^{\infty} \frac{\sin (n x)}{n^{2 N+1}}=\frac{(-1)^{N-1}}{2}(2 \pi)^{2 N+1} \phi_{2 N+1}\left(\frac{x}{2 \pi}\right)
$$

and

$$
\sum_{n=1}^{\infty} \frac{\cos (n x)}{n^{2 N}}=\frac{(-1)^{N-1}}{2}(2 \pi)^{2 N} \phi_{2 N}\left(\frac{x}{2 \pi}\right)
$$

where $\phi_{N}(x)$ is the $N$ th Bernoulli polynomial, normalized so that the high-order coefficient is $1 / N$ !, see [15, p. 430]. We divide (29) by $n^{7}$ and sum over $n$. Then, we would like to use (30) four times with $N=3$ and $x=1,3,5,7$. But there is a hitch: (30) is not valid for $x=7$ because $x>2 \pi$. So instead of 7 we use $7-2 \pi$. It is this value, $7-2 \pi$, substituted into the Bernoulli polynomial, that causes $s_{7}$ to be a 7 th degree polynomial in $\pi$. For $s_{13}$, for example, we would have to use $x=1,3,5,7-2 \pi$, $9-2 \pi, 11-2 \pi$, and $13-4 \pi$. For $N \geq 7$, we would end up with an $N$ th degree polynomial in $\pi$.

(c) With more effort this process yields a closed form for each such sum. First, for $N=7$ we have observed that

$$
-64 \sin ^{7}(n)=\sin (7 n)-7 \sin (5 n)+21 \sin (3 n)-35 \sin (n),
$$

and that

$$
\sum_{n=1}^{\infty} \frac{\sin (n x)}{n^{7}}=64 \pi^{7} \phi_{7}\left(\frac{x}{2 \pi}\right) \text { for } 0 \leq x \leq 2 \pi
$$

where

$$
\phi_{7}(x):=\frac{1}{30240} x-\frac{1}{4320} x^{3}+\frac{1}{1440} x^{5}+\frac{1}{5040} x^{7}-\frac{1}{1440} x^{6}
$$

is the Bernoulli polynomial of order seven. Note that in (32), 7 is the only coefficient that falls outside the interval $(0,2 \pi)$. Substituting (33) into (32) yields (25), provided 
instead of simply replacing $x$ with 7 , we replace $x$ with $7-2 \pi$ when dealing with the $\sin (7 n)$ term in (32), to stay in the interval where (33) is valid. The same procedure, with versions of (33) and (32) using cosines in place of sines, yields (26). An interesting additional computation shows that

$$
s_{7}+\frac{1}{2}-i_{7}=64 \pi^{7}\left\{\phi_{7}\left(\frac{7-2 \pi}{2 \pi}\right)-\phi_{7}\left(\frac{7}{2 \pi}\right)\right\} .
$$

In other words the difference between $s_{7}+1 / 2$ and $i_{7}$ resides in the one term in (32) with coefficient outside the interval $(0,2 \pi)$.

(d) Let us use the fractional part

$$
\{z\}_{2 \pi}:=\frac{z}{2 \pi}-\left\lfloor\frac{z}{2 \pi}\right\rfloor .
$$

In like fashion, we ultimately obtain pretty closed forms for each $s_{M}$.

For $M$ odd:

$$
s_{M}=\frac{(-1)^{\frac{M+1}{2}}}{M !} \pi^{M} \sum_{k=1}^{\frac{M+1}{2}}(-1)^{k+1}\left(\begin{array}{c}
M \\
\frac{M+1}{2}-k
\end{array}\right) \phi_{M}\left(\{2 k-1\}_{2 \pi}\right) .
$$

For $M$ even:

$$
s_{M}=\frac{(-1)^{M / 2}}{M !} \pi^{M} \sum_{k=0}^{\frac{M}{2}} \frac{(-1)^{k+1}}{\delta_{k, 0}+1}\left(\begin{array}{c}
M \\
\frac{M}{2}-k
\end{array}\right) \phi_{M}\left(\{2 k\}_{2 \pi}\right),
$$

where, as usual, $\delta_{k, 0}=1$ when $k=0$, and 0 otherwise. Remarkably, these formulae are rational multiples of $\pi$ exactly for $M \leq 6$ and thereafter are polynomials in $\pi$ of degree $M$.

Many variations on the previous themes are possible. For example, one may insert powers of cosine as in [5, Thm. 3], although it does not seem possible to extend Theorem 1 to this case. In simple cases it is, however, easy to proceed as follows:

Example 5. Let us introduce the notation

$$
s_{i, j}:=\sum_{n=1}^{\infty} \operatorname{sinc}(n)^{i} \cos (n)^{j} .
$$

We discovered experimentally that $s_{1,1}=s_{1,2}=s_{2,1}=s_{3.1}=s_{2,2}=\pi / 4-1 / 2$ and that in each case the corresponding integral equals $\pi / 4$. Likewise $s_{1.3}=s_{2.3}=s_{3,3}=$ $s_{1,4}=s_{2,4}=3 \pi / 16-1 / 2$ while the corresponding integrals are equal to $3 \pi / 16$. Except for $s_{1,2}, s_{2,2}, s_{2,3}$, and $s_{1,4}$, the identity $\operatorname{sinc}(n) \cos (n)=\operatorname{sinc}(2 n)$ allows us to apply Theorem 1. In the remaining four cases, we may use the method of Example 4 to prove the discovered results, but a good explanation has eluded us. Richard Crandall [9] has, however, recently pointed out that Poisson summation will produce a finite closed form for each $s_{i, j}$.

5. AN EXTREMAL PROPERTY. We finish with a useful Siegel-type lower bound, [2, Exercise 8.4], giving an extremal property of the sinc ${ }^{k}$ integrals. This has applications to giving an upper bound on the size of integral solutions to integer linear equations, [1]. In [1] it was intimated that the proof was easy; it appears not to be so: 
Theorem 3 (Lower Bound). Suppose $a_{0} \geq a_{k}>0$ for $k=1,2, \ldots, n$. Then

$$
\int_{0}^{\infty} \prod_{k=0}^{n} \operatorname{sinc}\left(a_{k} x\right) d x \geq \int_{0}^{\infty} \operatorname{sinc}^{n+1}\left(a_{0} x\right) d x .
$$

In view of Corollary 1 we then have the following:

Corollary 2. Suppose $a_{0} \geq a_{k}>0$ for $k=1,2, \ldots, n$ and $0<\tau A_{n}<2 \pi$. Then

$$
\begin{aligned}
\frac{\tau}{2}+\tau \sum_{r=1}^{\infty} \prod_{k=0}^{n} \operatorname{sinc}\left(\tau a_{k} r\right) & =\int_{0}^{\infty} \prod_{k=0}^{n} \operatorname{sinc}\left(a_{k} x\right) d x \\
& \geq \int_{0}^{\infty} \operatorname{sinc}^{n+1}\left(a_{0} x\right) d x
\end{aligned}
$$

Proof of Theorem 3. Let

$$
\tau_{n}:=\int_{0}^{\infty} \prod_{k=0}^{n} \operatorname{sinc}\left(a_{k} x\right) d x, \quad \mu_{n}:=\int_{0}^{\infty} \operatorname{sinc}^{n+1}\left(a_{0} x\right) d x,
$$

and, for $a>0$, let

$$
\chi_{a}(x):= \begin{cases}1, & \text { if }|x|<a \\ 1 / 2, & \text { if }|x|=a \\ 0, & \text { if }|x|>a\end{cases}
$$

Further, let

$$
F_{0}:=\frac{1}{a_{0}} \sqrt{\frac{\pi}{2}} \chi_{a_{0}}, \quad F_{n}:=(\sqrt{2 \pi})^{1-n} f_{1} * f_{2} * \cdots * f_{n},
$$

where

$$
f_{n}:=\frac{1}{a_{n}} \sqrt{\frac{\pi}{2}} \chi_{a_{n}}
$$

and $*$ indicates convolution, i.e.,

$$
f_{j} * f_{k}(x):=\int_{-\infty}^{\infty} f_{j}(x-t) f_{k}(t) d t .
$$

Then (see [5], and [12, p. 20, Entry 5.2]) $F_{0}$ is the Fourier transform of $\operatorname{sinc}\left(a_{0} x\right)$ and, for $n \geq 1, F_{n}$ is the Fourier transform of $\prod_{k=1}^{n} \operatorname{sinc}\left(a_{k} x\right)$. In addition, for $n \geq 1$, $F_{n}(x)$ is an even function which vanishes on $\left(-\infty,-\sigma_{n}\right] \cup\left[\sigma_{n}, \infty\right)$ and is positive on $\left(-\sigma_{n}, \sigma_{n}\right)$, where $\sigma_{n}:=A_{n}-a_{0}=a_{1}+a_{2}+\cdots+a_{n}$. Furthermore, for $n \geq 1, F_{n}(x)$ is monotone nonincreasing on $(0, \infty)$. Hence, by a version of Parseval's theorem (see [5]),

$$
\tau_{n}=\int_{0}^{\infty} F_{n}(x) F_{0}(x) d x=\frac{1}{a_{0}} \sqrt{\frac{\pi}{2}} \int_{0}^{\min \left(\sigma_{n}, a_{0}\right)} F_{n}(x) d x \text { for } n \geq 1 .
$$


Observe that, for $n \geq 2$,

$$
F_{n}=\frac{1}{\sqrt{2 \pi}} F_{n-1} * f_{n}
$$

and hence that, for $y>0$,

$$
\begin{aligned}
\int_{0}^{y} F_{n}(v) d v & =\frac{1}{\sqrt{2 \pi}} \int_{0}^{y} d v \int_{-\infty}^{\infty} F_{n-1}(v-t) f_{n}(t) d t \\
& =\frac{1}{2 a_{n}} \int_{0}^{y} d v \int_{-a_{n}}^{a_{n}} F_{n-1}(v-t) d t \\
& =\frac{1}{2 a_{n}} \int_{-a_{n}}^{a_{n}} d t \int_{0}^{y} F_{n-1}(v-t) d v \\
& =\frac{1}{2 a_{n}} \int_{-a_{n}}^{a_{n}} d t \int_{-t}^{y-t} F_{n-1}(u) d u
\end{aligned}
$$

Thus, we determine that

$$
\int_{0}^{y} F_{n}(v) d v=\int_{0}^{y} F_{n-1}(u) d u+I_{1}\left(a_{n}\right)+I_{2}\left(a_{n}\right),
$$

where, for $x>0$,

$$
I_{1}(x):=\frac{1}{2 x} \int_{-x}^{x} d t \int_{-t}^{0} F_{n-1}(u) d u \quad \text { and } \quad I_{2}(x):=\frac{1}{2 x} \int_{-x}^{x} d t \int_{y}^{y-t} F_{n-1}(u) d u
$$

Now $I_{1}(x)=0$ since $\int_{-t}^{0} F_{n-1}(u) d u$ is an odd function of $t$, and for $y \geq x$,

$$
\begin{aligned}
I_{2}(x) & =\frac{1}{2 x} \int_{0}^{x} d t \int_{y}^{y-t} F_{n-1}(u) d u+\frac{1}{2 x} \int_{-x}^{0} d t \int_{y}^{y-t} F_{n-1}(u) d u \\
& =\frac{1}{2 x} \int_{0}^{x} \phi(t) d t
\end{aligned}
$$

where

$$
\phi(t):=\int_{y}^{y+t} F_{n-1}(u) d u-\int_{y-t}^{y} F_{n-1}(u) d u \leq 0 \text { for } 0 \leq t \leq y,
$$

since $F_{n-1}(u)$ is monotonic nonincreasing for $u \geq 0$. Observe that $\phi^{\prime}(t)=$ $F_{n-1}(y+t)-F_{n-1}(y-t) \leq 0$ for $0 \leq t \leq y$, apart from at most two exceptional values of $t$ when $n=2$. Hence

$$
I_{2}^{\prime}(x)=\frac{1}{x^{2}} \int_{0}^{x}(\phi(x)-\phi(t)) d t=\frac{1}{x^{2}} \int_{0}^{x} d t \int_{t}^{x} \phi^{\prime}(u) d u \leq 0
$$

and so

$$
I_{2}(x) \text { is nonincreasing for } 0 \leq x \leq y .
$$


Our aim is to prove that $\tau_{n} \geq \mu_{n}$. Since, by Theorem 1 , this inequality automatically holds when $a_{0} \geq \sigma_{n}$, we assume that $a_{0}<\sigma_{n}$. Note that in case $n=1$ the hypothesis $a_{0} \geq a_{1}=\sigma_{1}$ immediately implies the desired inequality. Assume therefore that $n \geq 2$ in the rest of the proof. Suppose $a_{0}, a_{1}, \ldots, a_{n}$ are not all equal, and re-index them so that $a_{0}$ remains fixed and $a_{n}<a_{n-1} \leq a_{0}$. If $a_{n}$ is increased to $a_{n-1}$, it follows from (42) with $x=a_{n}$ and $y=a_{0}$ that $I_{2}\left(a_{n}\right)$ is not increased and hence, by (38), and (39) with $y=a_{0}$, that $\tau_{n}$ is not increased. Continuing in this way, we can coalesce all the $a_{k}$ 's into the common value $a_{0}$ without increasing the value of $\tau_{n}$. This final value of $\tau_{n}$ is, of course, $\mu_{n}$, and so the original value of $\tau_{n}$ satisfies $\tau_{n} \geq \mu_{n}$, as desired.

Perhaps a somewhat analogous version of Theorem 3 holds for sums?

ACKNOWLEDGEMENTS. J. M. Borwein's work is funded by NSERC and the Canada Research Chair Program.

\section{REFERENCES}

1. I. Aliev, Siegel's lemma and sum-distinct sets (preprint, 2005); available at http://arxiv.org/abs/ math/0503115v3.

2. D. Bailey, J. Borwein, N. Calkin, R. Girgensohn, R. Luke, and V. Moll, Experimental Mathematics in Action, A. K. Peters, Natick, MA, 2007.

3. R. J. Baillie et al., Solutions to advanced problem 6241, this MONTHLY 87 (1980) 497-498.

4. R. P. Boas and H. Pollard, Continuous analogues of series, this Monthly 80 (1973) 18-25.

5. D. Borwein and J. M. Borwein, Some remarkable properties of sinc and related integrals, Ramanujan J. 5 (2001) 73-90.

6. D. Borwein, J. M. Borwein, and B. Mares, Multi-variable sinc integrals and volumes of polyhedra, Ramanujan J. 6 (2002) 189-208.

7. J. M. Borwein and D. H. Bailey, Mathematics by Experiment: Plausible Reasoning in the 21 st Century, A. K. Peters, Natick, MA, 2004. Combined Interactive CD version, 2006.

8. J. M. Borwein, D. H. Bailey, and R. Girgensohn, Experimentation in Mathematics: Computational Paths to Discovery, A.K. Peters, Natick, MA, 2004. Combined Interactive CD version, 2006.

9. R. E. Crandall, Note on sinc-kernel sums and Poisson transformation (preprint, 15 June 2007). Available at http://www.reed.edu/ ${ }^{\sim}$ crandall.

10. R. E. Crandall, Theory of ROOF walks (preprint, 23 May 2008). Available at http://www.reed.edu/ crandall.

11. R. Crandall and C. Pomerance, Prime Numbers: A Computational Perspective, 2nd ed., Springer-Verlag, Heidelberg, 2005.

12. F. Oberhettinger, Tables of Fourier Transforms and Fourier Transforms of Distributions, Springer-Verlag, New York, 1991.

13. Mark A. Pinsky, Introduction to Fourier Analysis and Wavelets, Brooks/Cole, Pacific Grove, CA, 2002.

14. N. J. A. Sloane, The On-Line Encyclopedia of Integer Sequences. Available at http://www. research. att.com/ ${ }^{\sim}$ jas /sequences.

15. K. R. Stromberg, An Introduction to Classical Real Analysis, Wadsworth, Belmont, CA, 1981.

16. E. C. Titchmarsh, Introduction to the Theory of Fourier Integrals, Oxford University Press, Oxford, 1937.

ROBERT BAILLIE received B.S. and M.S. degrees in mathematics from the University of Illinois in Urbana in 1970 and 1971. Since then, he has worked as a software engineer, but maintains an interest in computational number theory, numerical analysis, and experimental mathematics.

Remcom, Inc., State College, PA

rjbaillie@frii.com

DAVID BORWEIN obtained two B.Sc. degrees from Witwatersrand University, one in engineering in 1945 and the other in mathematics in 1948. From University College London (UK) he received a Ph.D. in 1950 and a D.Sc. in 1960. He has been at the University of Western Ontario since 1963 with an emeritus title since 1989. His main area of research has been classical analysis, particularly summability theory.

Department of Mathematics, The University of Western Ontario, London, ONT, N6A 5B7, Canada dborwein@uwo.ca 
JONATHAN M. BORWEIN received his B.A. from the University of Western Ontario in 1971 and a D.Phil. from Oxford in 1974, both in mathematics. He currently holds a Canada Research Chair in the Faculty of Computer Science at Dalhousie University. His primary current research interests are in nonlinear functional analysis, optimization, and experimental (computationally-assisted) mathematics.

Faculty of Computer Science and Department of Mathematics, Dalhousie University,

Halifax NS, B3H 2W5, Canada

jborwein@cs.dal.ca

\section{A Very Short Evaluation of an Integral}

In [1], the author used partial fractions to evaluate the integral of an odd power of $\sec \theta$. Here, we give a one-sentence evaluation of this integral:

$$
\begin{aligned}
\int \sec ^{2 n+1} \theta d \theta= & \int \frac{\sec ^{2 n+1} \theta(\sec \theta+\tan \theta)}{\sec \theta+\tan \theta} d \theta \\
= & \int\left(\frac{t+1 / t}{2}\right)^{2 n} \frac{d t}{t} \quad(t:=\sec \theta+\tan \theta) \\
= & \frac{1}{2^{2 n}} \sum_{r=0}^{2 n}\left(\begin{array}{c}
2 n \\
r
\end{array}\right) \int t^{2 r-1-2 n} d t \\
= & \frac{1}{2^{2 n}}\left(\begin{array}{c}
2 n \\
n
\end{array}\right) \log t+\frac{1}{2^{2 n}} \sum_{r=0}^{2 n}\left(\begin{array}{c}
2 n \\
r \neq n
\end{array}\right) \frac{t^{2 r-2 n}}{2 r-2 n}+C \\
= & \frac{1}{2^{2 n}}\left(\begin{array}{c}
2 n \\
n
\end{array}\right) \log [\sec \theta+\tan \theta] \\
& +\sum_{r=0}^{n-1}\left(\begin{array}{c}
2 n \\
r
\end{array}\right) \frac{(\sec \theta+\tan \theta)^{2 n-2 r}-(\sec \theta-\tan \theta)^{2 n-2 r}}{2^{2 n+1}(n-r)}+C .
\end{aligned}
$$

In the second step, we have used the fact that $1 / t=\sec \theta-\tan \theta$, and in the last step, for each $r$ between 0 and $n-1$ we have combined the terms of the sum for $r$ and $2 n-r$. One can, of course, rewrite the expression in terms of $1 \pm \sin \theta$ easily because

$$
(\sec \theta+\tan \theta)^{2}=\frac{(1+\sin \theta)^{2}}{1-\sin ^{2} \theta}=\frac{1+\sin \theta}{1-\sin \theta} .
$$

\section{REFERENCE}

1. D. J. Velleman, Partial fractions, binomial coefficients, and the integral of an odd power of $\sec \theta$, this MonTHLY 109 (2002) 746-749.

- Submitted by B. Sury, Indian Statistical Institute, Bangalore, India 